\title{
Erratum to: Modified Vortex-Assisted Surfactant-Enhanced Emulsification Microextraction Using Methyl Benzoate for the Preconcentration of Fungicides in Fruit Juice Samples and Analysis by High-Performance Liquid Chromatography
}

Yanawath Santaladchaiyakit ${ }^{a, *, * *}$, Jittaya Sadchaiyaphum ${ }^{a}$, Nusara Phannorit ${ }^{a}$, Chutikarn Lekyoung , Nipaporn Chumwangwapee ${ }^{a}$, and Supalax Srijaranai ${ }^{b}$

${ }^{a}$ Department of Chemistry, Faculty of Engineering, Rajamangala University of Technology Isan, Khon Kaen Campus, Khon Kaen, 40000 Thailand

${ }^{b}$ Materials Chemistry Research Center, Department of Chemistry and Center of Excellence for Innovation in Chemistry, Faculty of Science, Khon Kaen University, Khon Kaen, 40002 Thailand

*e-mail: sanyanawa@gmail.com

**e-mail: yanawath.sa@rmuti.ac.th

Submitted April 1, 2020; accepted for publication April 1, 2020

DOI: $10.1134 /$ S1061934820070199

(1) Figure 2, legend $x$-axis should read as follows: Concentration of SDS, $\mathrm{mmol} / \mathrm{L}$

(2) Page 1031, the last sentence of Section "Evaluation of preconcentration factor and extraction recovery" should read as follows:
Calculated PF and ER were found to be in the range of $15-38$ and $31-77 \%$.

The original article can be found online at

https://doi.org/10.1134/S1061934819100095 